\title{
Order of Magnitude Reasoning in Qualitative Differential Equations
}

\author{
Ernest Davis
}

\begin{abstract}
We present a theory that combines order of magnitude reasoning with envisionment of qualitative differential equations. Such a theory can be used to reason qualitatively about dynamical systems containing parameters of widely varying magnitudes. We present an a mathematical analysis of envisionment over orders of magnitude, including a complete categorization of adjacent pairs of qualitative states. We show how this theory can be applied to simple problems, we give an algorithm for generating a complete envisionment graph, and we discuss the implementation of this algorithm in a running program.
\end{abstract}

30 October 2008 


\title{
Order of Magnitude Reasoning in Qualitative Differential Equations
}

\author{
Ernest Davis
}

\section{Introduction}

Two mathematical techniques that have been found particularly useful in recent work on qualitative physical reasoning are the solution of qualitative differential equations through envisionment and order of magnitude reasoning. The object of this paper is to combine these two theories mathematically.

Envisionment and qualitative differential equations are used to analyze the behavior of dynamical systems. If the physical structure of a dynamic system can be characterized in terms of relations between a finite set of state variables and their derivatives, then the envisionment process can determine at least partially the behavior over time of these variables and derivatives. The strength of the envisionment procedure is that it can use a partial categorization of the physical relations among the variables to derive a useful partial information about the behavior. For example, consider a block attached to a spring, moving in a straight line without friction. Given the weak constraint that the spring always exerts a force on the block pointed toward its rest point, the envisionment process can predict that the block will oscillate around the rest point. Envisionment has been applied in numerous physical domains, including electronics, mechanics, hydraulics, and heat transfer ([de Kleer and Brown, 85], [Forbus, 85], [Williams, 85]). The clearest exposition of the mathematics of the theory is [Kuipers, 85]. Envisionment suffers from a number of limitations: it is sometimes too weak mathematically to yield important inferences ([Kuipers, 85], [Struss, 87]), and its focus on differential behavior sometimes forces much complex but useless information to be generated ([Davis, 86]). Nonetheless, for many types of physical inference, it is a simple and effective mode of analysis.

Order of magnitude reasoning is concerned with the analysis of physical systems in which one quantity is much greater than another, or in the comparison of two systems of the same structure, but which have corresponding quantities of very different magnitudes. For example, if a very massive block hits a very light one, order of magnitude reasoning can be used to infer that the massive block continues on its way unaffected, while the light block bounces off away from the massive one. The idea of order of magnitude reasoning is to approximate a very great ratio as an infinite ratio, and then to analyze the system in terms of an algebra of infinite and infinitesimal quantities. Such an algebra has been worked out in [Raiman, 86] and extended in [Dague, Raiman, and Deves, 87].

The natural next step is to combine these two modes of inference so that we may reason about the dynamical behavior of systems with quantities of widely varying magnitudes. For example, we would like to be able to reason that a very heavy block on a spring will have a much longer period of oscillation than a much lighter block on the same spring. This paper presents a theory combining order of magnitude reasoning with envisionment of qualitative differential equations, which supports such inferences. The theory introduces two technical innovations. First, each state of the system is labelled with its duration and with the net change to each parameter during the state. Second, we formulate a number of rules governing the behavior of functions over orders of magnitudes. In other respects, each of our component subtheories is weaker than standard theories in the literature. Our theory of envisionment allows only the fixed quantity spaces of sign and order of magnitude, not arbitrary discretizations of quantity spaces as in [Kuipers, 85]. Our order of magnitude algebra uses only three fixed ranges of orders of magnitudes, SMALL, MEDIUM, and LARGE, not arbitrarily many as in [Raiman, 86] and [Dague, Raiman, and Deves, 87]. ([Weld, 87] presents an alternative method for making this inference, which does not assume that the ratio between the blocks is infinite, but rather does a careful analysis of the relation between changes in in parameters and their derivatives. This theory is stronger than ours, in that it requires weaker assumptions in the input, but it

The problem addressed here was first brought to my attention by Dan Weld. I thank Dan Weld, Olivier Raiman, Asher Meth, Ben Kuipers, Leo Joskowicz, and Yumi Iwasaki for helpful discussions, and for their criticisms of an preliminary draft of this paper. This research was supported by NSF grant DCR-8603758. 
seems to be less generally applicable.)

The paper is organized as follows: Section 2 presents fundamental definitions and rules that govern the behavior of a solution to a set of qualitative differential equations. The basis of the definitions and the justifications of the rules lie in the theory of non-standard analysis with infinitesimals. Section 3 shows how these rules may be applied in the "heavy block on the spring" example and other examples. Section 4 presents an algorithm for constructing an envisionment graph from a set of qualitative differential equations. Section 5 discusses the CHEPACHET program, which implements this algorithm. Section 6 makes suggestions for further work. We assume that the reader is familiar with standard theories of envisionment, as in [Kuipers, 85] and [de Kleer and Brown, 85], and with non-standard analysis, as in [Robinson, 66] and [Davis and Hersch, 72].

\section{Theory}

We follow [Kuipers, 85] in structuring our theory.

Quantities, derivatives, and time may take values from within the non-standard real line $R *$. We divide this line into seven disjoint intervals: (We use notation from [Dague, Raiman, and Deves].)

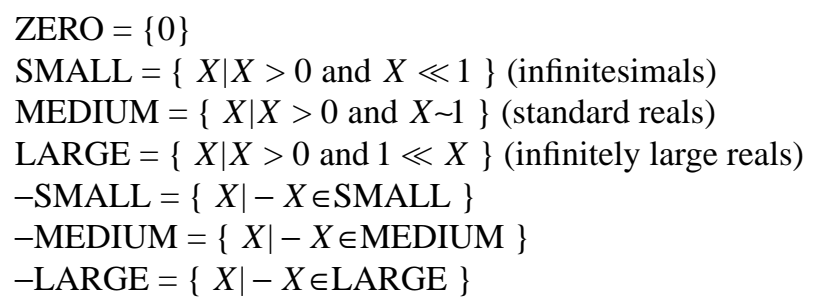

These sets and their unions are the qualitative sets. Note that SMALL and LARGE span many orders of magnitude while MEDIUM spans only one order of magnitude. Note also that there is no landmark value separating SMALL from MEDIUM or MEDIUM from LARGE; in non-standard analysis, there is no largest infinitesimal or smallest positive standard real. Our theory can easily be extended to incorporate any finite number of orders of magnitude; we might, for example, have five positive orders VERY_SMALL, SMALL, MEDIUM, LARGE, VERY_LARGE. It is not clear how to extend the theory to infinitely many different orders, as in [Dague, Raiman, and Deves, 87].

Table 1 shows the basic arithmetic operations on the qualitative values. These follow directly from the axioms presented in [Raiman, 86]. Following the notation of [de Kleer and Brown, 85] and [Kuipers, $85]$ we define $[X]$ to be the qualitative set containing the value $X$; for example $[-1]=-$ MEDIUM, $[0]=$ ZERO. If $P(T)$ is a function of $T$, then we will use the notation $\partial P$ for the qualitative value of the derivative; that is, $\partial P(T)=[\dot{P}(T)]$.

An interval of $R *$ is a set of points $I$ such that, for all $X, Y \in I$, if $X<Z<Y$ then $Z \in I$. An interval $I$ is closed on the left (right) if $I$ has a greatest lower bound (least upper bound) $X$, and $X$ is in $I$. It is open on the left (right) if it has a greatest lower bound (least upper bound) $X$, and $X$ is not in $I$ or if it is unbounded on the left (right). Thus, the interval ZERO is closed on both left and right; SMALL is open on the left and neither open nor closed on the right; MEDIUM is neither open nor closed on either side; and LARGE is neither open nor closed on the left and open on the right. Two intervals $I$ and $J$ are adjacent if they are disjoint and $I \cup J$ is an interval.

We will be interested in tracing the qualitative values of a number of parameters over time $p_{1}(T), p_{2}(T), \cdots p_{k}(T)$ and their derivatives $\dot{p}_{1}(T), \dot{p}_{2}(T), \cdots \dot{p}_{k}(T)$. The parameters and time take their values from the non-standard real line. The parameters are assumed to be $C^{1}$; that is, they are continuous, and their derivatives are continuous. A collection of $k$ such functions is called a behavior.

We define the inertial state of the parameters $p_{1}(T) \cdots p_{k}(T)$ to be the $2 k$-tuple of the qualitative states of all the parameters and their derivatives:

$$
S=\left\langle\left[p_{1}(T)\right] \cdots\left[p_{k}(T)\right],\left[\dot{p}_{1}(T)\right] \cdots\left[\dot{p}_{k}(T)\right]\right\rangle
$$

An inertial state $S$ characterizes a behavior $P=<p_{1}(T) \cdots p_{k}(T)>$ over a time interval $I$ if $S$ is the inertial state of $P$ for all $T$ in $I$. 
An inertial subdivision of an interval $I$ by a behavior $P$ is a set of subintervals of $I$, where $I_{\alpha} \in$ iff the following conditions hold:

i. $\quad I_{\alpha} \subseteq I$

ii. There is a single inertial state $S_{\alpha}$ that characterizes $P$ throughout $I_{\alpha}$.

iii. $\quad I_{\alpha}$ is maximal with respect to properties (i) and (ii).

It should be clear that, for any interval $I$ and behavior $P$, there is a unique subdivision; that the intervals of the subdivision are disjoint; and that their union is $I$.

We are also interested in the order of magnitude of intervals and of the change to a parameter over an interval. Let $P=<p_{1}(T) \cdots p_{k}(T)>$ be a behavior and let $I$ be a time interval. We define the time duration of $I$, written $\Delta T(I)$, to be the maximum over all points $T_{1}, T_{2} \in I$ of $\left[T_{2}-T_{1}\right]$. We define the variance of parameter $p(T)$ over $I$, written $\Delta p(I)$, to be the maximum over all $T_{1}, T_{2} \in I$ of $\left[p\left(T_{1}\right)-p\left(T_{2}\right)\right]$

A specification of the qualitative values $\left[p_{i}\right], \partial p_{i}, \Delta p_{i}$, and $\Delta T$ is called a qualitative state. We can now state a number of basic rules that relate the qualitative states of adjacent intervals in an inertial decomposition. Let $P=<p_{1}(T) \cdots p_{k}(T)>$ be a behavior; let $p$ be a parameter in $P$; let $I$ be an interval; and let $A$ and $B$ be two adjacent intervals in the inertial subdivision of $I$ by $P$. Then the following rules hold:

Change: There is a parameter $p$ such that either $[p(A)] \neq[p(B)]$ or $\partial p(A) \neq \partial p(B)$. That is, something changes from $A$ to $B$.

Continuity: From $A$ to $B,[p]$ and $\partial p$ must either stay constant, or go from one qualitative set to a bordering set, in the ordering -LARGE, -MEDIUM, -SMALL, ZERO, SMALL, MEDIUM, LARGE. (Note that variances and time durations do not have to change continuously.)

Mean value from ZERO: If $[p]=\mathrm{ZERO}$ in $A$ and $\partial p>0$ in $A$, then $[p]=$ SMALL in $B$. If $[p]=$ ZERO in $A$ and $\partial p<0$ in $A$, then $[p]=-$ SMALL in $B$.

Mean value to ZERO: If [ $p$ ] =ZERO in $B$ and $\partial p<0$ in $B$, then [ $p$ ]=SMALL in $A$. If [ $p$ ] =ZERO in $B$ and $\partial p>0$ in $B$, then $[p]=-$ SMALL in $A$.

Mean value from non-zero: If $[p]$ is greater in $B$ than in $A$, and is not equal to ZERO in either state, then $\partial p$ must be positive in both $A$ and $B$. If [ $p]$ is less in $B$ than in $A$, and is not equal to ZERO in either state, then $\partial p$ must be negative in both $A$ and $B$.

Variance over time: The variance of $p$ in $A$ is the absolute value of the derivative of $p$ times the duration.

$$
\Delta p \subseteq \operatorname{abs}(\partial p) \times \Delta T
$$

Variance bound: The variance of a parameter is never greater than its absolute value.

$$
\Delta p \leq \operatorname{abs}([p])
$$

Variance at state change: If $[p]$ changes from $A$ to $B$ then $\Delta p(A)=\operatorname{abs}([p(B)])$, and $\Delta p(A)=\operatorname{abs}([p(B)])$ Note the contrapositive of this statement: If $\Delta p$ in $A$ is small as compared to $[p]$ in $A$, then $[p]$ does not change from $A$ to $B$.

No successive instants: $A$ and $B$ cannot both have duration ZERO.

Temporal topology: By the rule of continuity, any transition of a parameter or derivative $p$ from $A$ to $B$ has one of the following forms:

i. $\quad p(A)=$ ZERO and $p(B)= \pm$ SMALL;

ii. $\quad p(A)= \pm$ SMALL and $p(B)=$ ZERO;

iii. $\quad p(A)= \pm$ MEDIUM and $p(B)= \pm$ SMALL or \pm LARGE;

iv. $\quad p(A)= \pm$ SMALL or \pm LARGE and $p(B)= \pm$ MEDIUM;

The rule of temporal topology states that, going from one interval $A$ to the next interval $B$, only one of these types of transitions can occur. Thus, for example, it is possible to have $p$ change from SMALL in $A$ to MEDIUM in $B$ and have $q$ change from -LARGE in $A$ to -MEDIUM in $B$, since these are both transitions of type (iv). It would not be possible to have $p$ change from SMALL in $A$ to MEDIUM in $B$, and $q$ 
change from ZERO in $A$ to SMALL in $B$, since the first is a transition of type (iv), while the second is a transition of (i).

The fundamental reason for this rule is that different types of transitions imply different topologies for the intervals. If a transition of type (i) occurs from $A$ to $B$, then $A$ must be closed on the right, and $B$ must be open on the left. If a transition of type (ii) occurs, then $A$ must be open on the right and $B$ must be closed on the left. If a transition of type (iii) or (iv) occurs, then $A$ must be neither open nor closed on the left, and $B$ must be neither open nor closed on the right. We can show that transitions of type (iii) cannot occur together with transitions of type (iv) by the following argument: Suppose that $p(T)$ goes from MEDIUM in $A$ to LARGE in $B$, and that $q(T)$ goes from SMALL in $A$ to MEDIUM in $B$. Then the continuous function $p(T) \cdot q(T)$ would go from SMALL in $A$ to LARGE in $B$, which is impossible.

Forever is a long time: If $A$ is unbounded on the left or on the right, so that it has no adjacent following state or no adjacent preceding state, then $\Delta T(A)=$ LARGE.

The rules of change, continuity, mean value, and no successive instants are the same or direct analogues of standard rules from the ordinary theory of QDE's ([Kuipers, 85], [Williams, 85]). William's "Epsilon Transition" rule, that a transition to zero must take finite time, is a consequence of the rules above: If $p$ is non-zero in $A$ and zero in $B$, then by the rule of "Variance at state change," $\Delta p$ is non-zero in $A$, so by the rule of variance over time, $\Delta T$ cannot be zero in $A$.

These rules together are a sufficient characterization of order of magnitude of values, derivatives, variances and durations in adjecent intervals. That is, given two qualitative states $S_{A}$ and $S_{B}$ satisfying the above rules, other than the "Forever" rule, one can find a behavior $P$ and two adjacent intervals $A$ and $B$, such that $S_{A}$ is the qualitative state of $P$ in $A$, and $S_{B}$ is the qualitative state of $P$ in $B$. Given a state $A$ satisfying the rules of "Variance over time", "Variance bound" and "Forever", there is a behavior which stays with qualitative state $A$ over all time. We omit the proof of this; it involves a long but straightforward case analysis, enumerating all the possible values of any parameter over the two states, constructing particular functions that exhibit each of them, and showing that any pair of transitions allowed together by the "Transition topology" rule will occur simultaneously for our sample functions.

A stronger hypothesis seems plausible, but we have no proof:

Hypothesis 1: Given any finite sequence of qualitative states such that any two successive qualitative states satisfy the above rules, there exists a behavior and an interval such that the qualitative states characterize the behavior over the interval.

So far, we have been speaking about the space of all possible behaviors. For any particular problem, we will be working with qualitative constraints on the problem. A homogeneous system of first-order qualitative constraints (abbreviated QC's) is a set of relations among the orders of magnitudes of the parameter values and their derivatives. ("Homogeneous" here refers to the absence of an explicit time dependence.) That is, such a system specifies certain combinations of the values $\left[p_{i}(T)\right]$ and $\partial p_{i}(T)$ to be physically possible, and others to be impossible. For example, we might specify the relation $\partial X=-[X]$; this states that the only qualitative states allowed are $[X]=$ LARGE, $\partial X=-$ LARGE; $[X]=$ MEDIUM, $\partial X=-$ MEDIUM, etc. A behavior $P$ satisfies a set of QC's only if the orders of magnitude of its parameters and their derivatives satisfy the set at each instant of time.

Thus, if a behavior satisfies a set of QC's, then all its inertial states satisfies the QC's, and any two adjacent qualitative states satisfy the rules enumerated above. If Hypothesis 1 above is true then the converse of this statement is likewise true:

Hypothesis 2: Given a set of QC's and a finite sequence of qualitative states, if each successive pair of states satisfies the rules above and the inertial state of each qualitative state satisfies the QC's, then there exist a behavior $P$ and a time interval $I$ such that the qualitative states characterize $P$ over $I$, and such that $P$ satisfies the QC's throughout $I$.

Proof given hypothesis 1: Use hypothesis 1 to construct a behavior $P$ and an interval $I$ following the qualitative states. Then $P$ satisfies the QC's since the states do.

A particularly important class of qualitative constraints are the qualitative differential equations (abbreviated QDE's). Let $F_{1}, \cdots, F_{k}$ be $k$ continuous $k$-place functions over the non-standard real line. Then the system of (exact) differential equations 


$$
\begin{gathered}
\dot{p}_{1}(T)=F_{1}\left(p_{1}(T), p_{2}(T) \cdots p_{k}(T)\right) \\
\dot{p}_{2}(T)=F_{2}\left(p_{1}(T), p_{2}(T) \cdots p_{k}(T)\right) \\
\cdots \\
\dot{p}_{k}(T)=F_{k}\left(p_{1}(T), p_{2}(T) \cdots p_{k}(T)\right)
\end{gathered}
$$

is guaranteed to have $C^{1}$ solutions for any given initial values $p_{1}(0) \cdots p_{k}(0)$. We now discretize the functions $F_{i}$ to multi-valued functions $G_{i}$ over qualitative sets. That is, if $Q_{1} \cdots Q_{k}$ are qualitative values, we define $G_{i}\left(Q_{1} \cdots Q_{k}\right)$ by considering all possible choices of $x_{1} \cdots x_{k}$ from $Q_{1} \cdots Q_{k}$, and taking the qualitative value of $F_{i}\left(x_{1} \cdots x_{k}\right)$.

$$
G_{i}\left(Q_{1} \cdots Q_{k}\right)=\left\{\left[F_{i}\left(x_{1} \cdots x_{k}\right)\right] \mid x_{1} \in Q_{1} \cdots x_{k} \in Q_{k}\right\}
$$

Thus, for example, if $F(X, Y)=X^{2}+Y$ then $G$ (SMALL,MEDIUM) $=$ SMALL $^{2}+$ MEDIUM $=$ $\{$ MEDIUM $\} . G($ MEDIUM, - MEDIUM $)=$ MEDIUM $^{2}-$ MEDIUM $=\{-$ MEDIUM, - SMALL, ZERO, SMALL, MEDIUM\}.

The condition that the $F_{i}$ be continuous implies conditions on the $G_{i}$ analogous to the rule of continuity and the rule of temporal topology given above.

A set of QDE's is then the discretized form of the original exact differential equations.

$$
\begin{gathered}
\partial p_{1}(T) \in G_{1}\left(\left[p_{1}(T)\right] \cdots\left[p_{k}(T)\right]\right) \\
\partial p_{2}(T) \in G_{2}\left(\left[p_{1}(T)\right] \cdots\left[p_{k}(T)\right]\right) \\
\cdots \\
\partial p_{k}(T) \in G_{k}\left(\left[p_{1}(T)\right] \cdots\left[p_{k}(T)\right]\right)
\end{gathered}
$$

Note that there can be behaviors that satisfy some system of QDE's but do not satisfy any system of homogeneous exact differential equations. For instance, any solution of a set of homogeneous exact differential equations that goes through the values $\langle 0,0,0 \ldots 0\rangle$ more than once must be cyclic, by the uniqueness of solutions to the initial value problem; this does not hold for solutions to homogeneous QDE's. 


\section{Sample Inferences}

We now show how these rules can be used to prove that a heavy block on a spring will take a long time to reach the rest point.

Let $x$ be the displacement of the block from its rest point, let $v$ be its velocity, and let $f$ be the force exerted by the spring on the block. The physical constraints on the system can be characterized by the following QDE's

$$
\begin{gathered}
\partial x=[v] \\
{[f]=\mathrm{LARGE} \cdot \partial v} \\
{[f]=-[x]}
\end{gathered}
$$

The first equation just establishes $v$ as the derivative of $x$. The second equation corresponds to Newton's second law $f=m a$. In this case, $m$ is assumed to be LARGE, and $a=\partial v$. The third equation is a very weak form of Hooke's law; it states that the spring exerts a force opposite to the displacement and of the same order of magnitude. ${ }^{1}$ For convenience of exposition, we will eliminate $f$ and rewrite the equations in the equivalent form:

$$
\begin{gathered}
\partial x=[v] \\
\partial v=[x] / \text { LARGE }
\end{gathered}
$$

Nothing in our analysis depends on this rewriting; it would go through in the same way in either presentation.

We now track the sequence of qualitative states consistent with the QDE's and with the rules enumerated above, and we show that any path which brings the block to its rest point includes a state whose duration is LARGE.

We start with the block at some finite displacement and zero velocity. That is, in state A1, $[x]=$ MEDIUM, $[v]=$ ZERO. From the differential equations, $\partial x=v$ must be ZERO, while $\partial v=-x /$ LARGE must be -SMALL. By variance bound, $\Delta v$ must be ZERO. By variance over time, therefore, $\Delta T=\frac{\Delta v}{|\partial v|}=$ ZERO. Applying variance over time in the other direction, $\Delta x=\Delta T \cdot \partial x=$ ZERO. So we have a complete description of A1:

$$
\begin{array}{rlrl}
\mathrm{A} 1: & {[x]=\text { MEDIUM }} & \partial x=\text { ZERO } & \Delta x=\text { ZERO } \\
{[v]=\text { ZERO }} & \partial v=- \text { SMALL } & \Delta v=\text { ZERO } \\
\Delta T=\text { ZERO } & &
\end{array}
$$

Let A2 be the next state. Since $[x]=$ MEDIUM and $\Delta x=$ ZERO in A1, by variance at state change $[x]$ must be MEDIUM in A2. By mean value from zero and continuity, $[v]$ must be -SMALL. From the differential equations, $\partial x$ and $\partial v$ must both be -SMALL. We cannot determine the variances or duration until we look at the next state.

Let $\mathrm{A} 3$ be the next state. In A3, either $[x],[v], \partial x$ or $\partial v$ must change. In fact, since the differential equations determine $\partial x$ and $\partial v$ from $[x]$ and $[v]$, either $[x]$ or $[v]$ must change. By mean value at non-zero and continuity, we must either have $[v]$ change to-MEDIUM, or have $[x]$ change to SMALL or both. We will consider each of these in turn:

Suppose that $[v]$ changes to -MEDIUM in A3. Then, by the rule of variance at state change, we must have $\Delta v=$ MEDIUM in A3. As we have stated, $[x]$ must either MEDIUM or SMALL in A3. Hence, from the differential equation $\partial v=[x] /$ LARGE must be -SMALL in A3. By variance over time, in A3, $\Delta T=\frac{\Delta v}{\partial v \mid}=$ LARGE. But, from the differential equations, $\partial x=[v]=$ MEDIUM, and, by variance over time, $\Delta x=\Delta T \cdot|\partial x|=$ LARGE. But this contradicts the rule of bounded variance, since $\Delta x$ is now larger than $[x]$. Thus the assumption that $[v]$ changes to -MEDIUM must have been false.

1. It may be asked, what does it mean to compare the order of magnitude of a force with that of a distance?. The answer is that we are assuming here standard units of mass, distance, and time. Thus, the question being addressed is actually a comparative one: assuming the mass is large compared to a standard mass, what does that imply about the time, compared to a standard time? 
The only option left for A3, therefore, is that $[x]$ changes to SMALL, and that $[v]$ remains -SMALL. From the differential equations, we have $\partial x=-$ SMALL, and $\partial v=-$ SMALL. Applying the variance at state change to A2 and A3, we must have that in A2, $\Delta x=$ MEDIUM, and in A3, $\Delta x=$ SMALL. Therefore, by variance over time in A2, we have $\Delta T=\frac{\Delta x}{|\partial x|}=$ LARGE. Combining bounded variance and variance over time, we find that, in A2, $\Delta v=$ SMALL. We have now completely characterized A2: 


$$
\begin{array}{llll}
\mathrm{A} 2: & {[x]=\text { MEDIUM }} & \partial x=- \text { SMALL } & \Delta x=\text { MEDIUM } \\
& {[v]=- \text { SMALL }} & \partial v=- \text { SMALL } & \Delta v=\text { SMALL } \\
\Delta T=\text { LARGE } & &
\end{array}
$$

Thus, we have shown that, before the block can reach the zero point, it must pass through a state of LARGE duration, which was the desired result.

We cannot show in this system that the block ever will reach the rest point. Since $\Delta T=$ LARGE in A2, it is consistent with our rules that the system should stay in A2 forever. In fact, the behavior

$$
\begin{gathered}
x(T)=1+e^{-\delta T^{2}} \\
v(T)=\delta\left(e^{-\delta T}-1\right)
\end{gathered}
$$

where $\delta$ is a SMALL real number, starts in $\mathrm{A} 1$ at $\mathrm{T}=0$, and then stays in $\mathrm{A} 2$ forever. (See figure 1.)

Figure 1: A behavior that stays in A2 forever

By contrast, solutions to the corresponding second order QDE $[\ddot{x}(T)]=-[x(T)] /$ LARGE cannot stay in A2 forever; $x(T)$ must cross the value ZERO. We can make the following argument by contradiction. Suppose $x(T)$ is always positive. Then $\ddot{x}(T)$ will always be negative, so $\dot{x}(T)$ will be uniformly decreasing. Thus, if $\dot{x}(T)$ attains a value $-\varepsilon$ at some time $T 1$, it will have value less than or equal to $-\varepsilon$ for all $T>T 1$. However, for $T>T 1$,

$$
x(T)-x(T 1)=\int_{T 1}^{T} \dot{x}(T) d T<-\varepsilon(T-T 1)
$$

Thus $x(T)$ will be negative for all $T>T 1+x(T 1) / \varepsilon$, contradicting the assumption. Thus, information is irrecoverably lost in converting the higher-order QDE into a first-order QDE. (Nothing in this argument depends on the order of magnitude mechanism. The same loss of information happens in converting the ordinary second-order QDE $[\ddot{x}(T)]=-[x(T)]$ to the two first order QDE's $\partial x(T)=[v(T)], \partial v(T)=-[x(T)]$.

Our rules also do not allow us to say much after what happens to the system past this state. In fact, from this starting state, the system can reach any consistent intertial state whatever, except for the rest state $[x]=$ ZERO, $[v]=$ ZERO .

In a similar way, the rules above allow us to show results such as the following:

Fast exponential growth and decay: The initial value problem (IVP) $\partial x=\operatorname{LARGE}[x]$, $[x(0)]=$ MEDIUM, spends a SMALL time with $[x]=$ MEDIUM, and then goes to $[x]=$ LARGE forever. The problem $\partial x=-\operatorname{LARGE}[x],[x(0)]=$ MEDIUM, spend a SMALL time with $[x]=$ MEDIUM and then goes to $[x]=$ SMALL. It then either stays forever with $[x]=$ SMALL, or it transits after a period of undetermined length to $[x]=$ ZERO, where it stays forever.

Slow exponential growth and decay: The IVP $\partial x=\operatorname{SMALL}[x],[x(0)]=$ MEDIUM, spends a LARGE time with $[x]=$ MEDIUM, and then goes to $[x]=$ LARGE forever. The problem $\partial x=-$ SMALL $[x],[x(0)]=$ MEDIUM, spend a LARGE time with $[x]=$ MEDIUM and then goes to $[x]=$ SMALL. It then either stays forever with $[x]=$ SMALL, or it transits after a period of undetermined length to $[x]=$ ZERO, where it stays forever.

Highly damped oscillation: The IVP 


$$
\begin{array}{ll}
\partial x=[v] & {[x(0)]=\text { MEDIUM }} \\
\partial v=-\operatorname{LARGE}[v]-[x] & {[v(0)]=\text { SMALL }}
\end{array}
$$

corresponding to a very heavily damped harmonic oscillator, transits immediately to $[x]=$ MEDIUM, $[v]=$ SMALL. It stays thus for a LARGE time, ( $\partial v$ can in the meantime take on a wide range of values) and eventually transits to the state $[x]=$ SMALL $[v]=-$ SMALL, From here on in, it can oscillate indefinitely cyclically with the absolute values of both $x$ and $v$ remaining SMALL or ZERO, or it can settle forever in any such state, or in the state $[x]=[v]=$ ZERO.

Quickly settling control parameter: The IVP

$$
\begin{array}{ll}
\partial u(T)=-\operatorname{LARGE}[u(T)] & {[u(0)]=\text { MEDIUM }} \\
\partial x(T)=-(\operatorname{MEDIUM}+[u(T)]) \cdot x(T) & {[x(0)]=\text { MEDIUM }}
\end{array}
$$

represents a quickly settling function $u(T)$ controlling a more slowly moving function $x(T)$. The solution has $[u(T)]$ become SMALL or ZERO in SMALL time, while $x(T)$ remains MEDIUM. Thereafter, $x(T)$ undergoes exponential decay with a MEDIUM time constant, eventually getting to SMALL values after LARGE time. (It takes only MEDIUM time to get to any value in MEDIUM but LARGE time to get to any value in SMALL.) Such problems are similar to those studied by Kuipers (1987).

\section{Algorithm}

We next present an algorithm for constructing an envisionment graph for a given set of QDE's. An envisionment graph for a set of QDE's is a data structure that indicates the transitions possible for solutions of the QDE. If hypothesis 2 is correct, it thus indicates all the finite characterizations of parts of solutions. The value of envisionment graphs for this class of problems is somewhat limited, for two reasons. First, they are very large. Since all initial value problems have solutions, any assignment of qualitative values to the parameters must be a possible state of the system. Thus there are at least $7^{k}$ inertial states for a system of $k$ parameters; in general, there will be more, since some of the QDE functions $G_{i}$ will be multi-valued. In practice, this limits the generation of envisionments to QDE's with at most three variables.

Second, the characterization of a behavior over all time may be considerably richer than its behavior over any finite sequence of qualitative states. For example, consider the (second-order) IVP

$$
\begin{array}{lll}
{[\ddot{x}(T)]=-[x(T)]} & {[x(0)]=\text { MEDIUM }} & {[\dot{x}(0)]=\text { ZERO }} \\
{[\dot{u}(T)]=\text { MEDIUM }} & {[u(0)]=\text { MEDIUM }} &
\end{array}
$$

We would like to say that $x$ oscillates forever in MEDIUM amounts of time, while $u$ attains LARGE values over LARGE amounts of time. However, nothing about finite sequences will ever say this. In fact, there is no allowable transition in which $u$ goes from MEDIUM to LARGE.

In this case, of course, it is possible to separate the two equations, and give separate histories, along the lines suggested in [Williams, 86]. However, it is not clear how this can be done in general; for example, how it could be done for the equations

$$
\begin{array}{ll}
{[\ddot{x}(T)]=-[u(T)] \cdot[x(T)]} & {[x(0]=[\dot{x}(0)]=\text { SMALL }} \\
{[\dot{u}(T)]=\text { MEDIUM }+[x]} & {[u(0)]=\text { MEDIUM }}
\end{array}
$$

Despite these difficulties, we have studied the construction of envisionment graphs as a natural and simple first step toward deeper analysis. We will discuss possible directions for improvement in section 6 . (Forbus (1987) gives a defence of building envisionments for conventional QDE's. There, the first problem is much alleviated, since the number of qualitative values is smaller, and the second problem can be ruled out.)

We will construct an envisionment graph whose nodes are intertial states, labelled by sets of possible variances and durations, and whose directed arcs are transitions, labelled by sets of the possible variances and durations that the end nodes can take, if the transition occurs. For example, figure 2 shows a piece of a graph, with nodes $A$ and $B$, and a transition from $A$ to $B$. The labels on $A$ mean that $[X]$ is MEDIUM in $A$, $\partial X$ is MEDIUM, $\Delta X$ is either SMALL or MEDIUM, and $\Delta T$ is either SMALL or MEDIUM. The labels on $B$ mean that $[X]$ is LARGE in $B$, that $\partial X$ is SMALL, that $\Delta X$ is LARGE, and that $\Delta T$ is LARGE. The labels on the arc indicate that, if a behavior goes from $A$ to $B$, then it will have $\Delta X$ and $\Delta T$ equal to 
MEDIUM in $A$, and $\Delta X$ and $\Delta T$ equal to LARGE in $B$. 
Figure 2: A labelled transition

Algorithm 1 constructs an envisionment graph from a set of QDE's. It is clearly a sound algorithm; that is, if $x(t)$ is a possible solution to the QDE's for all $t$, then any transition of $x(t)$ is represented in the graph. Soundness holds since the algorithm does nothing more than enforce the starting QDE's and the rules governing transitions. If hypothesis 2 is correct, then the algorithm is also complete; that is, any path through the graph represents the behavior of some solution of the QDE's over an interval. This may be somewhat surprising, since constraint propagation is not, in general complete. However, the significance of labels in this graph is different than for most labelled graphs. If one goes around a cycle in the envisionment graph and comes back to the same node, it need not have the same value as before, since it corresponds to the behavior of the function at a different time. Therefore, in this context, local consistency of the graph is enough to establish its global consistency.

By indexing inertial states in an array by the tuple of parameter values, step 3 of the algorithm, which connects bordering states, can be made reasonably efficient. It does not involve comparing all pairs of inertial states, but only states that differ by 0 or \pm 1 in each of the $k$ parameter values and the $k$ derivatives. It can be shown that the procedure above runs in time at most quadratic in the number of inertial states (which is, of course, itself exponential in the number of variables.)

\section{The CHEPACHET Program}

The above algorithm has been implemented as a program named CHEPACHET. CHEPACHET is written in Franz Lisp and runs on a VAX/780. It is roughly 600 lines of code long, and contains 85 function definitions, not including utility macros and comments. It has not been compiled, and has not been particularly optimized for efficiency. Input to CHEPACHET is a list of parameters and a list of QDE's, whose left side is the derivative of a parameter, and whose right side is a polynomial in the parameters and the qualitative values. The output of CHEPACHET is a listing of the graph.

Table 2 shows the results of running CHEPACHET on a number of simple QDE's in terms of the CPU time used (in CPU seconds, exclusive of garbage collection time), and the size of the envisionment graph generated. Garbage collection time was typically one-quarter to one-third of the other CPU time.

\section{Further Work}

Clearly, the above analysis and the CHEPACHET programs are only first steps. The theory will have to be considerably refined before it is of any practical value. Some important issues to be addressed include the following:

1. How can this theory be used in a goal-directed way, so that useful information, such as illustrated in section 3, can be extracted without generating the whole envisionment graph? It would be relatively easy to fix the algorithm to generate only the states that can be attained from a given set of initial conditions, but that would not necessarily improve matters much. In the problem of the heavy brick on a spring, every state but one (the rest state) can be reached from the starting conditions. Many of the states generated in a complete envisionment, such as the SMALL values that always lie between MEDIUM and ZERO values, are not particularly significant for any kind of inference. Can they be suppressed?

2. As discussed in section 3, information is lost when a higher-order qualitative differential equation is converted into a system of first-order equations. Is there any easy way to extract that information working directly with the higher order equations? ([De Kleer and Bobrow, 84] is the only substantial study of higher-order QDE's)

3. How can behaviors involving infinitely many state transitions in bounded time be characterized?

4. Can the theory be extended in a reasonable way to handle more levels of orders of magnitude? Currently, once a parameter and its derivative both become SMALL or both become LARGE, it becomes impossible to say anything at all about their relative sizes. A more flexible representation might support much more powerful inferences. 


\section{References}

[Bobrow, 85] D. Bobrow, ed. Qualitative Reasoning about Physical Systems, MIT Press, 1985

[Dague, Raiman, and Deves, 87] P. Dague, O. Raiman, and P. Deves, "Troubleshooting: When Modeling is the Trouble," Proc. AAAI, 1987, pp. 600-605

[Davis, 86] E. Davis, "A Logical Framework for Solid Object Physics," NYU Tech. Rep. 245

[Davis and Hersh, 72] M. Davis and R. Hersh, "Nonstandard Analysis," Scientific American, June, 1972, pp. $78-84$

[de Kleer and Bobrow, 84] J. de Kleer and D. Bobrow, "Qualitative Reasoning with Higher-Order Derivatives," Proc. AAAI 1984, pp. 86-91

[de Kleer and Brown, 85] J. de Kleer and J.S. Brown, "A Qualitative Physics Based on Confluences," in [Bobrow, 85], pp. 7-84

[Forbus, 85] K. Forbus, "Qualitative Process Theory," in [Bobrow, 85], pp. 85-168

[Forbus, 87] K. Forbus, "The Qualitative Process Engine: A study in Assumption-based Truth Maintenance," presented at the Qualitative Physics Workshop, Urbana, Illinois, 1987.

[Kuipers, 85] B. Kuipers, "The Limits of Qualitative Simultation," Proc. IJCAI, 1985, pp. 128-136

[Kuipers, 87] B. Kuipers, "Abstraction by Time-Scale in Qualitative Simulation," Proc. IJCAI 1987, pp. $621-625$

[Raiman, 86] O. Raiman, "Order of Magnitude Reasoning," Proc. AAAI, 1986, pp. 100-104.

[Robinson, 66] A. Robinson, Nonstandard Analysis, North Holland, Amsterdam, 1966.

[Struss, 87] P. Struss, "Mathematical Aspects of Qualitative Reasoning," presented at the Qualitative Physics Workshop, Urbana, Illinois, 1987.

[Waltz, 75] D. Waltz, "Understanding line drawings of scenes with shadows," in P. Winston, ed. The Psychology of Computer Vision, McGraw-Hill, 1975.

[Weld, 87] D. Weld, "Comparative Analysis," Proc. IJCAI, 1987, to appear.

[Williams, 85] B. Williams, "Qualitative Analysis of MOS Circuits," in [Bobrow, 85], pp. 281-346

[Williams, 86] B. Williams "Doing Time: Putting Qualitative Reasoning on Firmer Ground," Proc. AAAI, 1986, pp. 105-112 